\title{
Gold/Chitosan Nanocomposites with Specific Near Infrared Absorption for Photothermal Therapy Applications
}

\author{
Guandong Zhang, ${ }^{1}$ Xinghua Sun, ${ }^{1}$ Jacek Jasinski, ${ }^{2}$ Dhruvin Patel, ${ }^{1}$ and Andre M. Gobin ${ }^{1}$ \\ ${ }^{1}$ Department of Bioengineering, Speed School of Engineering, University of Louisville, Louisville, KY 40292, USA \\ ${ }^{2}$ Conn Center for Renewable Energy Research and Speed School of Engineering, University of Louisville, Louisville, KY 40292, USA
}

Correspondence should be addressed to Andre M. Gobin, andre.gobin@louisville.edu

Received 5 May 2012; Revised 27 July 2012; Accepted 27 August 2012

Academic Editor: Gaurav Mago

Copyright ( 2012 Guandong Zhang et al. This is an open access article distributed under the Creative Commons Attribution License, which permits unrestricted use, distribution, and reproduction in any medium, provided the original work is properly cited.

\begin{abstract}
Gold/chitosan nanocomposites were synthesized and evaluated as a therapeutic agent for the photothermal therapy. Gold nanoparticles (Au NPs) with controllable optical absorption in the near infrared (NIR) region were prepared by the reaction of chloroauric acid and sodium thiosulfate. To apply these particles to cancer therapy, the bare Au NPs were coated with chitosan (CS), O-carboxymethyl chitosan (CMCS), and a blend of CS and CMCS for utilizations in physiologic conditions. The surface properties, optical stability, and photothermal ablation efficiency on hepatocellular carcinoma cells (HepG2) and human dermal fibroblast cells (HDF) demonstrate that these gold nanocomposites have great potential as a therapeutic agent in in vitro tests. The CS-coated nanocomposites show the highest efficiency for the photo-ablation on the HepG2 cells, and the CS and CMCS blended coated particles show the best discrimination between the cancer cell and normal cells. The well-controlled NIR absorption and the biocompatible surface of these nanocomposites allow low-power NIR laser activation and low-dosage particle injection for the cancer cell treatment.
\end{abstract}

\section{Introduction}

The use of nanoparticles (NPs) in biomedical field is one of the most important branches of nanobiotechnology. Many biomedical applications require nanoparticles with low toxicity, biocompatibility, and stability with high preferential accumulation in the target tissue or organ [1]. Polymernanoparticle composites may possess the unique property superior to that of either the polymer or nanoparticles. Metal nanoparticles with polymer coatings or dispersed in polymeric matrices display the increased stability, improved processability, recyclability, and solubility in a variety of solvents [2].

Gold nanoparticles (Au NPs) are attracting enormous attentions in applications for immunoassay [3, 4], drug delivery [5], contrast enhancement, and thermal therapy for tumor treatment [6-8], due to their nanoscale size, oxidefree components, bioconjugation property, biocompatibility, and unique optical properties.

Thermal therapy, also termed as hyperthermia, has thousands years history as one of most important methods for the tumor treatment [9]. In this technique, moderate heating is generated in the target region, resulting in tumors selectively destroyed, due to their reduced heat tolerance compared to normal tissue [10]. In the past decades, because many new heating sources such as radiofrequency [11], microwave [12], magnetic field [13], and laser [9] were employed, there were remarkable progresses achieved in this area. Photothermal therapy (PT) is a promising field within thermal therapy, incorporating laser techniques and photoabsorbing agents. Light absorbing dyes, chemical photosensitizers, and solid optically responsive nanomaterials have been applied as the photoabsorbing agents in PT [9]. For small metal nanoparticles, their optical properties are dominated by collective oscillation of conduction electrons. An absorption band results when the incident photon frequency is resonant with the collective oscillation of the conduction band electrons, and this is known as the surface plasmon resonance (SPR) [14]. Au NPs possess strongly enhanced visible and near infrared light absorption, which is several orders of magnitude more intense compared to conventional chromophores. Various gold-based nanostructures with NIR 
absorption have been developed. Gold nanospheres [15], gold nanoshells [7], gold nanorods [16], and gold nanocages [17] are the popular nanostructures that have been demonstrated in photothermal therapeutics due to their strongly enhanced absorption in the visible and NIR regions on account of their SPR effects.

In our work, Au NPs with NIR absorption were prepared by the one step reaction of chloroauric acid and sodium thiosulfate. Their NIR absorption wavelengths were well controlled by adjusting molar ratio of $\mathrm{HAuCl}_{4} / \mathrm{Na}_{2} \mathrm{~S}_{2} \mathrm{O}_{3}$ [18]. These $\mathrm{Au}$ NPs were coated with chitosan- (CS)based coatings and used as a therapeutic agent for the photothermal ablation of cancer cells. Chitosan is a biobased polysaccharide derived from shrimp and crab shells [19]. It is nontoxic, biodegradable and biocompatible, and has good processability $[20,21]$. The cost associated with chitosan is much lower than that of other polymer coatings or chemical compounds, for example, polyethylene glycol (PEG) that are often used to protect and stabilize the nanoparticles by many researchers [5]. Hepatocellular carcinoma is the most common primary malignant tumor of the liver. It affects greater than half a million patients worldwide. US liverrelated cancer deaths account for $4 \%$ of all cancers or about 20,000 deaths annually. Two anchorage-dependent cell lines, hepatocellular carcinoma cell line (HepG2), and control cell line of human dermal fibroblast (HDF) were selected for the estimation of laser ablation efficiency of these novel gold nanocomposites. The efficacy was evaluated by live/dead stain images following photothermal ablation. These gold nanocomposites show great ability for laser ablation, and the surface coating components plays a very important role for selectivity to certain cancer cells. In addition, this composite material offers new and desirable advantages for cancer treatment. Beside the optically tunable gold nanoparticle for efficient conversion of light to heat, chitosan-based coatings provide tunable surface charges for applications in a variety of physiological pH environments and also allow further modification with targeting molecules or drug molecules by chemical reaction or electrostatic interaction.

\section{Experimental}

2.1. Preparation of $A u$ Nanoparticles. Au NPs were prepared by the method we reported before $[18,22]$. In which, $3 \mathrm{mM}$ sodium thiosulfate $\left(\mathrm{Na}_{2} \mathrm{~S}_{2} \mathrm{O}_{3} \cdot 5 \mathrm{H}_{2} \mathrm{O}\right.$, Aldrich, $99.999 \%)$ solution was added into $1.71 \mathrm{mM}$ chloroauric acid $\left(\mathrm{HAuCl}_{4} \cdot 3 \mathrm{H}_{2} \mathrm{O}\right.$, Alfa Aesar, $\left.\mathrm{Au} 49.68 \%\right)$ solutions with desired volume ratio at room temperature and vortexed for 20 seconds for uniform mixing. All the water used in the experiments was purified by a Thermo Scientific Easypure II system, with a resistivity of $18.2 \mathrm{M} \Omega \mathrm{cm}$.

2.2. Preparation of Carboxymethylated Chitosan. Low molecule weight chitosan (Sigma-Aldrich, MW 50-190 K) was used for the surface coating of the Au NPs. Based on this chitosan, O-carboxymethyl chitosan (CMCS) was synthesized as the reported method $[23,24]$. Briefly, $15 \mathrm{~g}$ sodium hydroxide was dissolved in a 20:80 mixture of deionized water and isopropanol $(100 \mathrm{~mL})$ in a $500 \mathrm{~mL}$ flask. $10 \mathrm{~g}$ chitosan was added to the flask to swell and alkalize at $50^{\circ} \mathrm{C}$ for $1 \mathrm{~h} .15 \mathrm{~g}$ monochloroacetic acid $\left(\mathrm{ClCH}_{2} \mathrm{CO}_{2} \mathrm{H}, 99 \%\right.$, Acros Organics) was dissolved in $20 \mathrm{~mL}$ isopropanol and added into the reaction mixture dropwisely in $30 \mathrm{~min}$ and reacted under vigorous agitation for $4 \mathrm{~h}$ at $55-60^{\circ} \mathrm{C}$. Adding $200 \mathrm{~mL} 80 \%$ ethyl alcohol stopped the reaction. The product was filtered and repeatedly rinsed by $80 \%$ ethyl alcohol, to desalt and dewater, until the $\mathrm{pH}$ value of the washing solution was less than 8.0. Finally, the product was vacuum dried for 1 day at $40^{\circ} \mathrm{C}$.

\subsection{Preparation and Purification of Au NPs with Chitosan} Based Coatings. Stock solution of chitosan was prepared by dispersing chitosan ( $1 \mathrm{~g}$ ) in $0.7 \%$ acetic acid solution $(100 \mathrm{~mL})$. CMCS solution was prepared by directly dissolving CMCS $(1 \mathrm{~g})$ in $100 \mathrm{~mL}$ DI water. CS and CMCS solutions were purified by centrifuge at $1000 \mathrm{rpm}$ for $10 \mathrm{~min}$, to remove the little amount of insoluble residuals, and then were dialyzed (Spectrum Dialysis Membranes, MWCO $3 \mathrm{KDa}$ ) for 2 days. The chitosan-coated Au NPs (Au/CS), CMCS coated Au NPs (Au/CMCS), and CS and CMCS blended coated $\mathrm{Au}$ NPs (Au/(CS+CMCS)) were prepared by, respectively, adding CS solution, CMCS solution, or CS and CMCS blending solution (CS and CMCS solutions were premixed) dropwisely into the as-synthesized gold particle suspensions, with vigorous agitation. The ratio between CS or CMCS to Au NPs was controlled as $0.2 \mathrm{mg}$ CS or CMCS per OD Au NP suspension and between CS and CMCS blending to $\mathrm{Au}$ NPs is $(0.15 \mathrm{mg} \mathrm{CS}+0.05 \mathrm{mg}$ CMCS $)$ per OD Au NP suspension (OD is the optical density). Then these samples were set on the rocking bed for 1 day. In the end the gold nanocomposite suspensions were concentrated and separated from the solution by centrifugation at $1000 \mathrm{~g}$ for $20 \mathrm{~min}$. This step will remove the byproducts in solution and the extra chitosan. Then the centrifuged pellets were dispersed in sterilized DI water to a concentration of $25 \mathrm{OD} / \mathrm{mL}$ and stored at $4^{\circ} \mathrm{C}$ for the subsequent studies.

2.4. Characterization of $A u$ NPs. The optical absorption of $\mathrm{Au}$ NPs was measured by a UV-Visible spectrophotometer (Cary-50Bio, Varian). A Zetasizer (Nano-ZS90, Malvern) was used to assess the surface charge of the nanoparticle suspensions. A FEI Tecnai F30 transmission electron microscope (TEM) operated at $200 \mathrm{KV}$ was used to determine the morphology of Au NPs. Several Au NPs suspensions that have different NIR peak wavelength were carefully prepared for the photo-induced temperature rising measurement. $1 \mathrm{~mL}$ of the Au NP suspensions were added into the wells of tissue culture plate (Multiwell, Primaria 24 Well), with the concentration of $1 \mathrm{OD} / \mathrm{mL}$. A laser with $817 \mathrm{~nm}$ centered wavelength generated by FAT-System (Coherent, CA) was shone on the gold suspension. The laser spot has the same area with the well, and the power of the laser was fixed as $1 \mathrm{~W} / \mathrm{cm}^{2}$. An infrared thermometer (CSmicro, Optris $\mathrm{GmbH}$, Germany) detecting the temperature of the Au NP suspension was fixed $10 \mathrm{~cm}$ above the media.

2.5. Photothermal Ablation Study. All cell lines were obtained from ATCC (Manassas, MA). Human dermal fibroblasts cell 


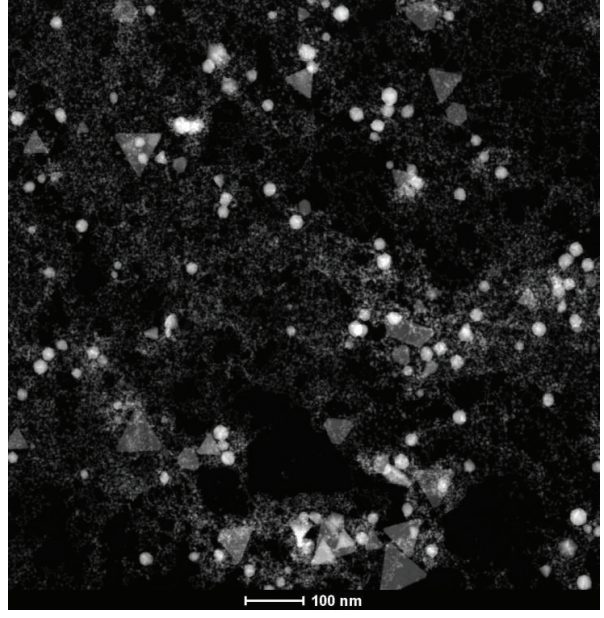

(a)

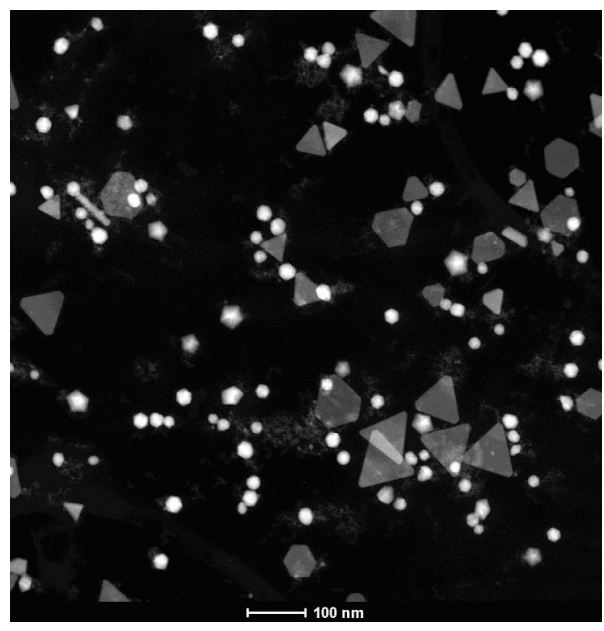

(b)

Figure 1: Z-contrast STEM images of the Au NPs synthesized from reaction of $\mathrm{HAuCl}_{4}$ and $\mathrm{Na}_{2} \mathrm{~S}_{2} \mathrm{O}_{3}$ before and after centrifugation process. (a) As-synthesized Au NPs; (b) Au NPs after purification by centrifugation. The purified product has the NIR absorption peak at $820 \mathrm{~nm}$.

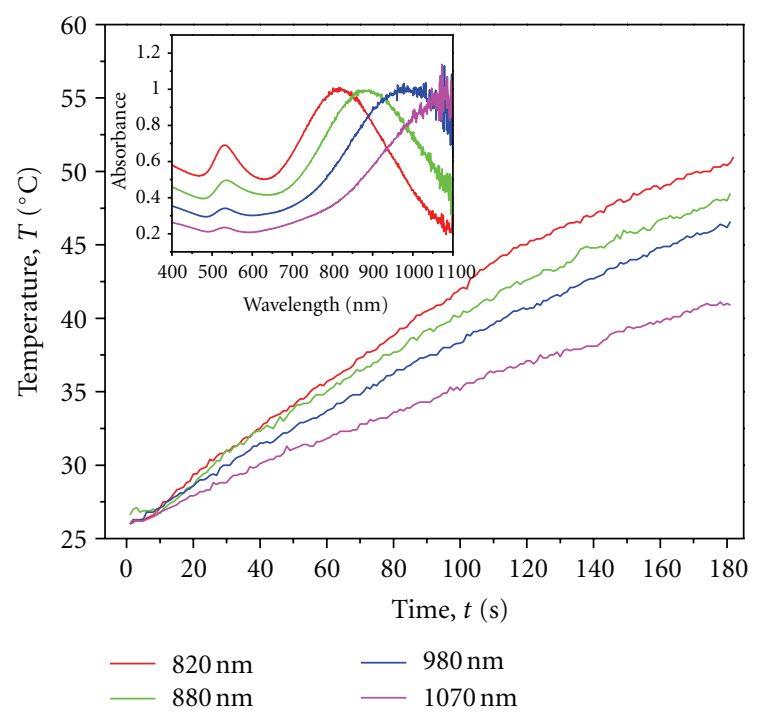

FIGURE 2: Heating effect of the gold nanoparticles with NIR absorption peaks at $820 \mathrm{~nm}, 880 \mathrm{~nm}, 980 \mathrm{~nm}$, and $1070 \mathrm{~nm}$, respectively, under the $817 \mathrm{~nm}$ wavelength laser exposures. The laser power is $1 \mathrm{~W} / \mathrm{cm}^{2}$, and the Au NPs were dispersed in water at a concentration of $1 \mathrm{OD} / \mathrm{mL}$. Inset shows the UV-visible-NIR spectra of the four $\mathrm{Au}$ NPs samples.

line (HDF, ATCC: PCS-201-012 male source) and hepatocellular carcinomas cell line (HepG2, ATCC: 59194) were seeded at monolayer confluence to allow for easier cell counting following staining (within 15 passages). All the cells were cultured at $37^{\circ} \mathrm{C}$ in a $5 \% \mathrm{CO}_{2}$ environment in Dulbecco's modified eagle medium (DMEM) supplemented with $4 \mathrm{mM}$ l-glutamine, $1 \%$ penicillin, and $10 \%$ fetal bovine serum. Prior to laser ablation experiments, cells were detached from culture flash with trypsin $(0.25 \%)$ and resuspended in DMEM media and injected into the 24-well tissue culture plate (Multiwell, Primaria), cultured for 36-48 hours to achieve confluence. Laser ablation was performed based on methods described in [7]. Cells were incubated with $1 \mathrm{~mL}$ DMEM media which contains 1 OD Au NPs for $2 \mathrm{~h}$ to obtain the binding of the Au NPs on the cell. For this experiment, $\mathrm{Au}$ NPs and gold/chitosan nanocomposites were prepared with NIR absorption in the range of $820 \mathrm{~nm}$ to $850 \mathrm{~nm}$, close to the wavelength of the laser source. Media were removed, and cells were rinsed with $0.5 \mathrm{~mL}$ PBS buffer, followed by addition of $0.5 \mathrm{~mL}$ fresh media to the culture wells. Next, the cells were exposed to $817 \mathrm{~nm}$ NIR laser at optimized conditions $\left(5 \mathrm{~W} / \mathrm{cm}^{2}, 2 \mathrm{~min}\right)$. Well plates were incubated overnight after photothermal treatment, and a live/dead stain (Invitrogen, Carlsbad, CA) was performed, and fluorescent images were obtained and analyzed. Images were taken with a Nikon D90 camera on an Accu-Scope 3032 Microscope for green/red fluorescence, respectively, and cell numbers were counted manually.

\section{Results and Discussion}

3.1. Preparation of Gold Nanoparticles with Desired Near Infrared Absorption. In our previous work [18, 25], a convenient method was developed to synthesize Au NPs with controllable NIR absorption. Au NPs were synthesized by one-step reaction of chloroauric acid and sodium thiosulfate, without assistance of additional templates, capping reagents, or seeds assembly. The synthesized products consist of nanoparticles with different shape and size including small spherical colloid gold particles $(<5 \mathrm{~nm})$ and nonspherical gold crystals. The nonspherical gold crystals are mainly the truncated octahedron, pentagons, and cuboctahedron, as well as the triangular-shaped plate structures [18]. Two absorption peaks due to the surface plasmon resonance are observed from these samples. The first SPR peak centered at around $530 \mathrm{~nm}$ is the characteristic SPR of the spherical colloid gold particles, and the second SPR component is higher at NIR band, which is attributed to the multipolar SPR band from the nonspherical Au NPs [18, 25]. Since the NIR absorption wavelengths increase with increasing 


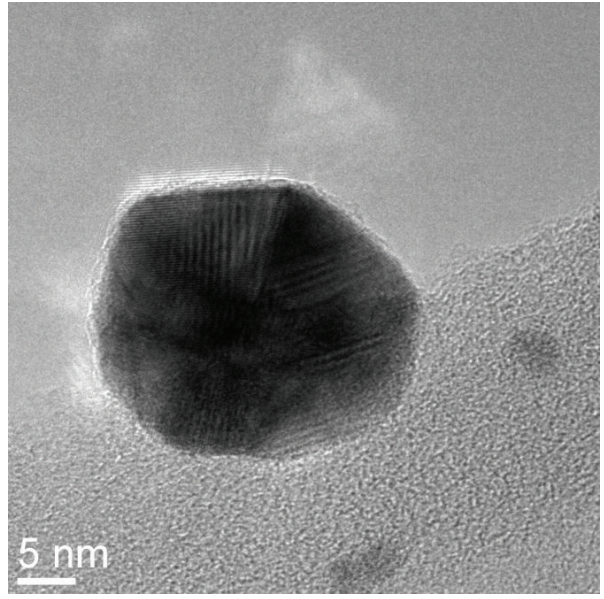

(a) Au Bare

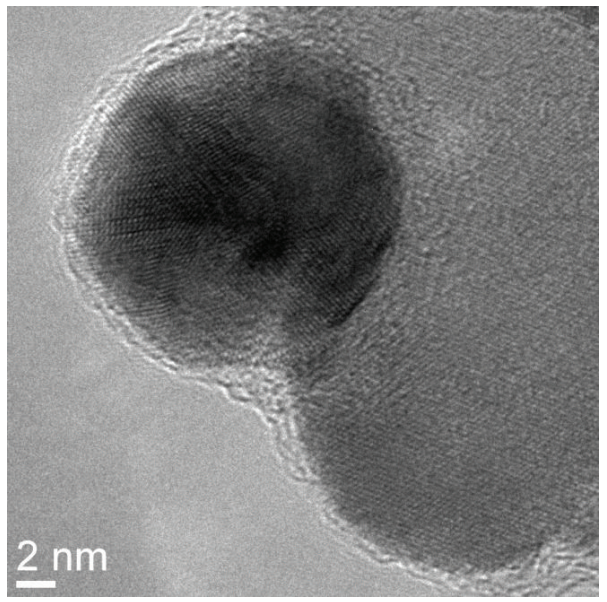

(c) $\mathrm{Au} / \mathrm{CMCS}$

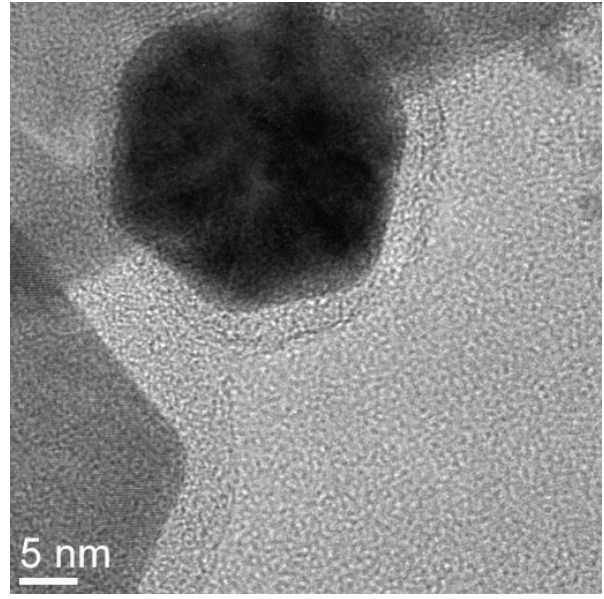

(b) $\mathrm{Au} / \mathrm{CS}$

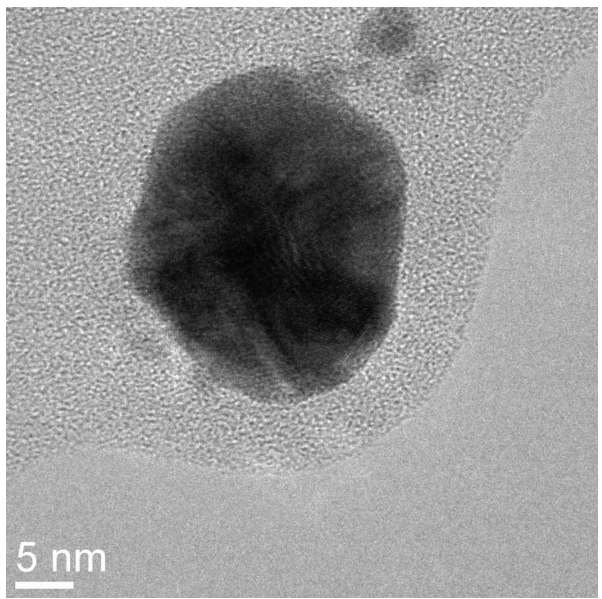

(d) $\mathrm{Au} /(\mathrm{CS}+\mathrm{CMCS})$

FIgure 3: High-resolution TEM images of the Au NPs: (a) Au NPs without coating, (b) Au/CS nanocomposite, (c) Au/CMCS nanocomposite, and (d) Au/(CS+CMCS) nanocomposite.

molar ratio of $\mathrm{HAuCl}_{4} / \mathrm{Na}_{2} \mathrm{~S}_{2} \mathrm{O}_{3}$, adjusting the molar ratio of $\mathrm{HAuCl}_{4} / \mathrm{Na}_{2} \mathrm{~S}_{2} \mathrm{O}_{3}$ during the reaction controlled the absorption wavelength of the Au NPs. These products were further purified by the centrifugation to remove the spherical colloid gold particles, improving the NIR absorption. Figures 1(a) and 1(b) are the Z-contrast STEM images of the assynthesized Au NPs and the same batch sample purified with centrifugation. The purified product possesses the NIR absorption wavelength at $820 \mathrm{~nm}$. The temperature of the NIR absorbing Au NP suspension induced by the NIR laser radiation is affected by the concentration of nanoparticles, laser power, exposure time, and the absorbing wavelength of the Au NPs [26]. Figure 2 compares the heating effect of the $\mathrm{Au}$ NPs suspensions with NIR peak at $820 \mathrm{~nm}, 880 \mathrm{~nm}$, $980 \mathrm{~nm}$, and $1070 \mathrm{~nm}$, respectively (showing in the insert figure) under the $817 \mathrm{~nm}$ wavelength diode laser $\left(1 \mathrm{~W} / \mathrm{cm}^{2}\right)$ exposures. After 3 minutes, the temperature reaches to 50.8, $48.2,46.4$, and $41.2^{\circ} \mathrm{C}$ for the particles from low wavelength to high wavelength, respectively. The gold sample that has the NIR peak more close to the wavelength of laser source shows the best heating effect. The effective control of the NIR absorption of the gold nanoparticles for matching the wavelength of the laser source enhances the hyperthermia treatment. Meanwhile, the NIR wavelength is considered as the best light source for the photothermal therapy, because it is minimally absorbed by the normal tissues components of water and hemoglobin and can transmit deeply in the body.

\subsection{Chitosan-Based Coatings on the Gold Nanoparticles.} Chitosan is a cationic polysaccharide of D-glucosamine and $\mathrm{N}$-acetyl-D-glucosamine and has been shown to be a highly biocompatible material and have antibiotic properties. To improve its solubility or achieve new functionality, chemical modifications of chitosan were conducted and many derivatives have been synthesized. For example, modifications can be synthesized by carboxylation of the hydroxyl or amine groups in chitosan structure [20], resulting in the $O$-carboxymethyl chitosan or $N$-carboxymethyl chitosan respectively. $\mathrm{O}$-carboxymethyl chitosan is achieved by substituting parts of the $-\mathrm{OH}$ at $\mathrm{C}-6$ position of glucosamine units with $-\mathrm{CH}_{2} \mathrm{COOH}$. Therefore, the reactive ligands such as $-\mathrm{COOH}$ and $-\mathrm{NH}_{2}$ groups are amenable to chemical functions. Others have reported the stabilization of GNPs with chitosan. As chitosan in solution is protonated and 


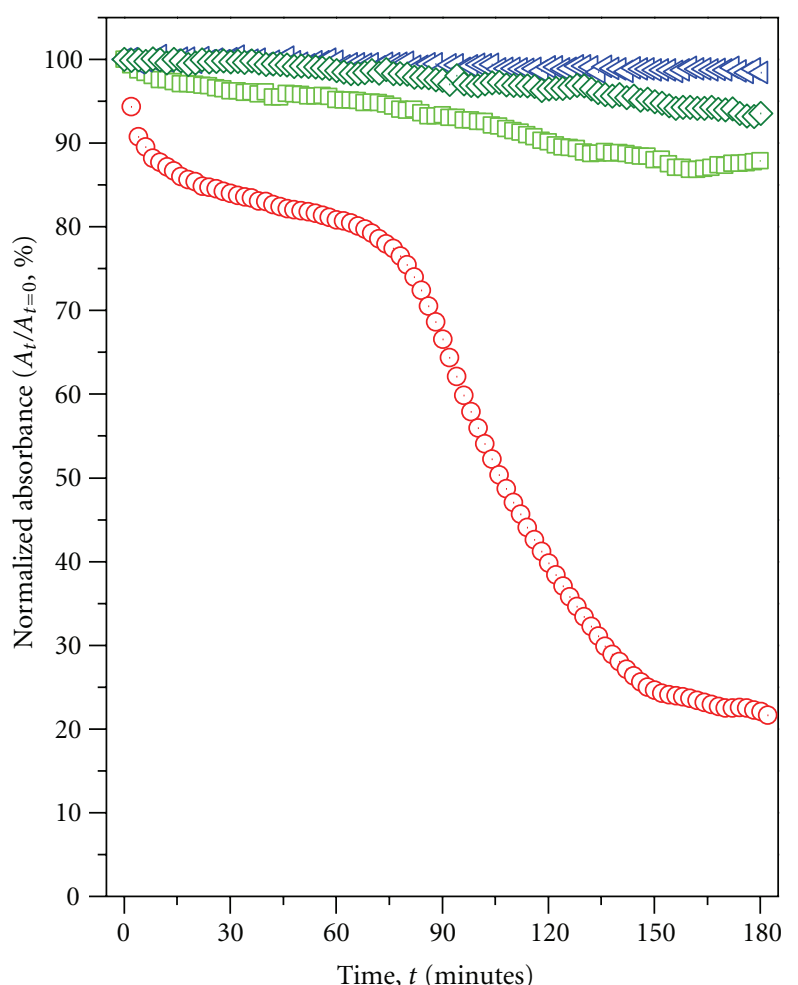

In 1X PBS

$\bigcirc$ Bare $\mathrm{Au}$

$\square \mathrm{Au} / \mathrm{CS}$

$\triangleleft \mathrm{Au} / \mathrm{CMCS}$

$\diamond \mathrm{Au} /(\mathrm{CS}+\mathrm{CMCS})$

(a)

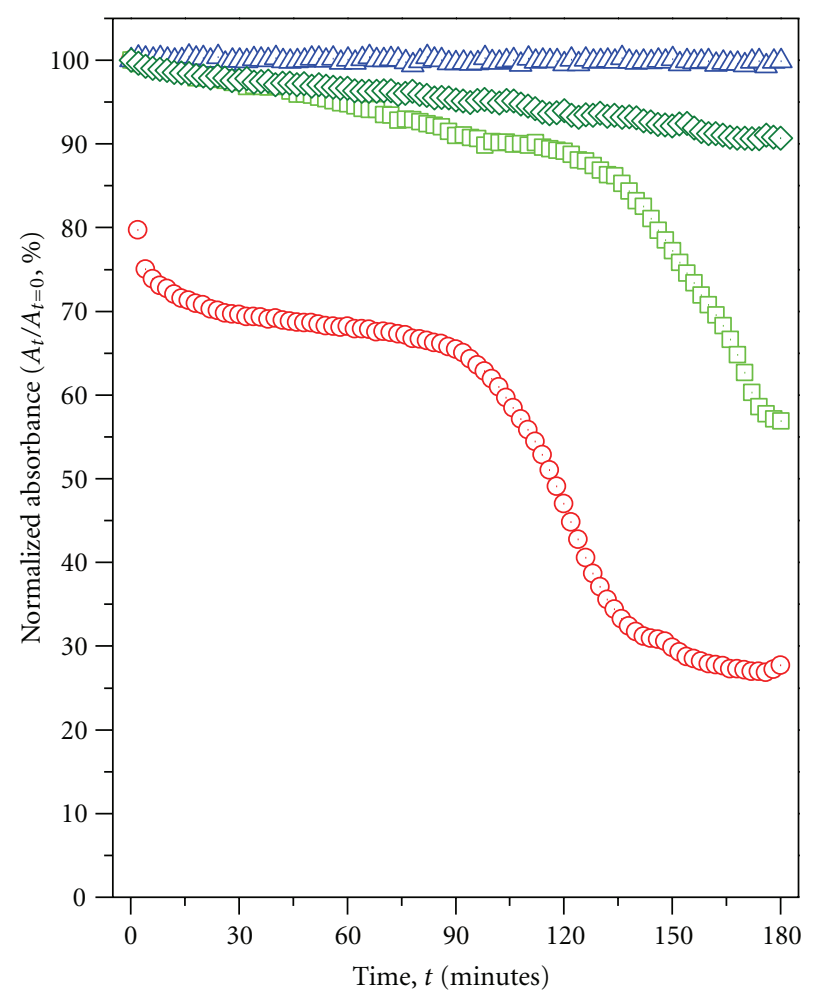

In 1 wt. $\% \mathrm{NaCl}$

$\begin{array}{ll}\bigcirc \mathrm{Bare} \mathrm{Au} & \diamond \mathrm{Au} / \mathrm{CMCS} \\ \square \mathrm{Au} / \mathrm{CS} & \diamond \mathrm{Au} /(\mathrm{CS}+\mathrm{CMCS})\end{array}$

(b)

FIGURE 4: Optical stability of the Au NPs with different chitosan coatings in (a) PBS solution and in (b) 1 wt.\% NaCl solution.

positively charged, it can be adsorbed onto the surfaces of gold nanoparticles, stabilizing and protecting the nanoparticles [27-29]. As-synthesized $\mathrm{Au}$ NPs present strongly negative surface charge (around $-40 \mathrm{mV}$ ) allowing the positive charged chitosan tightly coated onto gold surface via electrostatic interaction.

Figures 3(a), 3(b), 3(c), and 3(d) show the high-resolution TEM images of the Au NPs without coating, Au/CS, $\mathrm{Au} / \mathrm{CMCS}$, and $\mathrm{Au} /(\mathrm{CS}+\mathrm{CMCS})$ nanocomposites, respectively. The as-synthesized bare Au NPs have a 1-2 nm thin layer on their surface. Comparing to the bare Au NPs, Figures 3 (b) -3 (d) show the chitosan-based coatings effectively cover the gold NPs uniformly, forming a $2-5 \mathrm{~nm}$ thick layer on the gold surface.

Table 1 lists the hydrodynamic diameter and the surface charge of the gold nanoparticles with different chitosanbased coatings in water. The chitosan-based coating significantly changed the surface property of the gold nanoparticles. The average particle size of the bare gold nanoparticles is $36 \mathrm{~nm}$; after being coated with polymer, the hydrodynamic diameter of $\mathrm{Au} / \mathrm{CS}, \mathrm{Au} / \mathrm{CMCS}$, and $\mathrm{Au} /(\mathrm{CS}+\mathrm{CMCS})$ reaches 290, 236, and $262 \mathrm{~nm}$, respectively. Meanwhile, their surface charge changes from $-43.5 \mathrm{mV}$ to $+43.8,+20.2$ and $+24.3 \mathrm{mV}$, respectively.

Figure 4 shows the optical stability of the Au NPs with different coatings in PBS and $1 \mathrm{wt} \% \mathrm{NaCl}$ solution. Since the physiologic salinity is near $1 \%$, optical properties of $\mathrm{Au}$ NPs were simulated in these solutions. The optical stabilities were measured by monitoring the optical absorbance of the Au NPs at their NIR peak wavelength as function of time. These nanoparticles have similar behavior in the PBS and $\mathrm{NaCl}$ solution. CMCS-coated particles show the highest stability, and there is no obvious decrease in its optical absorption in $3 \mathrm{~h}$. The CS and CMCS blended coated particles also show good stability, with a decrease in its absorbance less than $10 \%$. The most unstable particles are the bare Au NPs. Its absorbance decreases more than $70 \%$ in saline environment. The Au/CS nanocomposites are more stable than the bare particles, but less stable than the Au NPs with CMCS component in the coating. The decrease of NIR absorption of the bare Au NPs results from interparticle coupling effect $[30,31]$, in which its stabilized surface layer was disturbed due to the high ion strength, causing the particle aggregation (irreversible coalescence). This behavior may greatly decrease the efficiency of photothermal ablation. Chitosan-based coatings greatly improve the optical stability of the Au NPs. The instability of the Au/CS NPs mainly results from the sedimentation of particles caused by the agglomeration (reversible coalescence) of chitosan coating. At the $\mathrm{pH}$ value above 6 , the solubility of chitosan becomes worse. CMCS has the best solubility in all $\mathrm{pH}$ ranges except its isoelectrical point [23]. The good solubility and the 
TABLE 1: Surface property of the gold nanoparticles with different chitosan-based coatings. Particles are dispersed in DI water with a concentration of $1 \mathrm{OD} / \mathrm{mL}$.

\begin{tabular}{lccr}
\hline Nanoparticles & Coating components & Hydrodynamic diameter $(\mathrm{nm}$, mean \pm STD $)$ & Zeta Potential $(\mathrm{mV}, \mathrm{mean} \pm$ STD $)$ \\
\hline Au Bare & None & $36.0 \pm 1.78$ & $-43.5 \pm 2.92$ \\
$\mathrm{Au} / \mathrm{CS}$ & $\mathrm{CS}$ & $290.4 \pm 52.7$ & $+43.8 \pm 4.36$ \\
$\mathrm{Au} / \mathrm{CMCS}$ & $\mathrm{CMCS}$ & $235.5 \pm 9.05$ & $+20.2 \pm 0.68$ \\
$\mathrm{Au} / \mathrm{CS}+\mathrm{CMCMS})$ & 75 wt. $\%$ CS + & $262.3 \pm 58.9$ & $+24.3 \pm 0.80$ \\
\hline
\end{tabular}

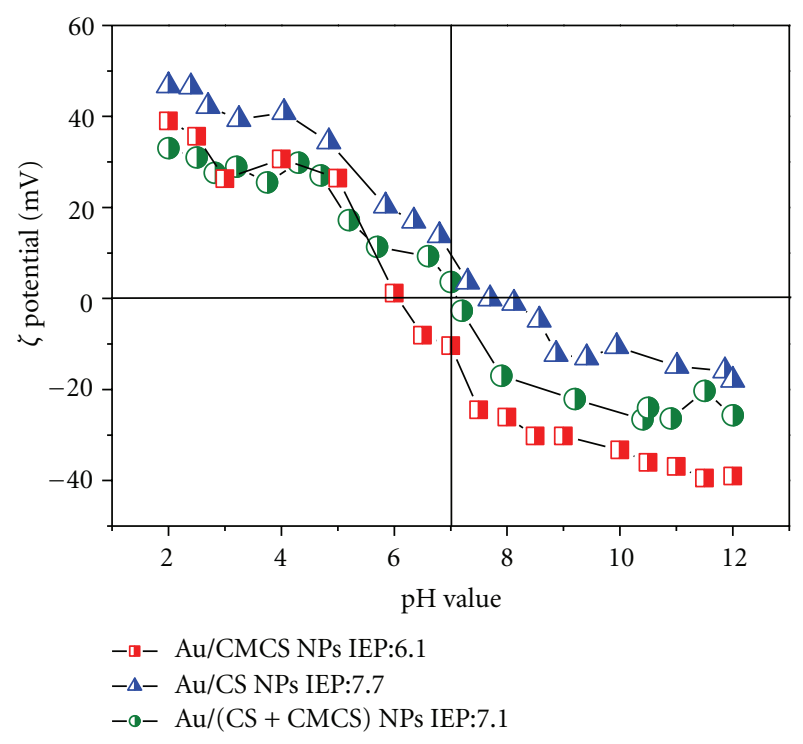

Figure 5: Effect of $\mathrm{pH}$ on Zeta potential of Au NPs with different coatings. Square point: Au/CMCS NPs, Triangle point: Au/CS NPs, Sphere point: $\mathrm{Au} /(\mathrm{CS}+\mathrm{CMCS}) \mathrm{NPs}$.

increased chain flexibility [32] of CMCS may prevent the coupling effect and the sedimentation of the particles.

By adjusting the surface components, the surface charge of the Au NPs can be modified. Figure 5 shows the zeta potential of $\mathrm{Au}$ NPs with different chitosan coatings as function of the $\mathrm{pH}$ value. The isoelectric points (IEPs) of $\mathrm{Au} / \mathrm{CMCS}, \mathrm{Au} /(\mathrm{CS}+\mathrm{CMCS})$, and $\mathrm{Au} / \mathrm{CS}$ nanocomposites are $6.1,7.1$, and 7.7, respectively. IEP of CMCS is close to value (5.5) reported by Zhao et al. [33]. CMCS presents amine and carboxylic acid groups in its structure. In solution, the groups turn to the ion state, $-\mathrm{NH}_{3}{ }^{+}$and $-\mathrm{COO}^{-}$, respectively, which improves the solubility of CMCS. Only at its IEP, the number of $-\mathrm{NH}_{3}{ }^{+}$is equal to that of $-\mathrm{COO}^{-}$, resulting in the deceasing of solubility. Au/CS particle shows much higher IEP value than that of the Au/CMCS particles, since the CS only presents the positively charged groups $\left(-\mathrm{NH}_{3}{ }^{+}\right)$. The IEP of the $\mathrm{Au} /(\mathrm{CS}+\mathrm{CMCS})$ particle is 7.1, between the those of Au/CMCS and Au/CS. This shows that the surface charge of Au NPs can be tuned by the chitosan components on their surface, since CMCS possesses more negatively charged characteristic than CS.

3.3. Photothermal Ablation of the HepG2 Cells. Figures 6 and 7 show the live/dead stain images of the HDF and HepG2 cells after being exposed to the $817 \mathrm{~nm}$ NIR laser at $5 \mathrm{~W} / \mathrm{cm}^{2}$, $2 \mathrm{~min}$. The live/dead stain provides a two-color fluorescence assay of cell viability based on plasma membrane permeability, for reliably and quantitatively distinguishing live and dead cells. The live cells show the green color and dead cells show the red color. The statistical cell death ratio of HDF cells and HepG2 cells counted from the live/dead stain images are shown in Figure 8.

Without incubation with Au NPs, cells do not exhibit obvious ablation after the laser irradiation at $5 \mathrm{~W} / \mathrm{cm}^{2}$, $2 \mathrm{~min}$, as shown in Figures 6(b) and 7(b). The bare Au NPs do not show significant photo-destruction on either cell lines (Figures 6(c) and 7(c)), due to the interparticle coupling effect [31]. The relatively low photothermal ablation efficiency of Au/CMCS NPs is probably due to their less adsorption on the cell membrane (Figures 6(e) and 7(e)). The highest efficiency of the photothermal ablation is observed from the cells treated with Au/CS NPs. There are almost 100\% HepG2 cells, and 90\% HDF cells were killed after the laser treatment (Figures 6(d) and 7(d)). The CS and CMCS blended coated gold NPs present the promising result: around 96\% HepG2 cancer cells were ablated and less than 30\% HDF normal cells died (Figures 6(f) and 7(f)). The differences of the Au NPs in the photothermal ablation result from the density of the Au NPs adsorbed on the cell surface. The negatively charged cell surface allows $\mathrm{Au} / \mathrm{CS}$ NPs to highly accumulate on the cell surface, and high density particles generate extraordinary heat, damaging the HepG2 cells as well as the HDF cells under the laser radiation. $\mathrm{Au} /(\mathrm{CS}+\mathrm{CMCS})$ particles bind to the cell surface uniformly and generate suitable heat, which was high enough to kill the cancer cells but not enough to damage all the normal cells. This consists with laser ablation results by using gold nanorod [16]. It was reported that after exposure to continuous red laser, malignant cells require about half the laser energy to be photothermally destroyed than the nonmalignant cells [16].

\section{Conclusions}

Gold/chitosan nanocomposites were synthesized for the photothermal therapy applications. Au NPs were prepared by the reaction of chloroauric acid with sodium thiosulfate, and they have specific NIR absorption matching the wavelength of laser source. These Au NPs were coated with chitosan or O-carboxymethyl chitosan as well as a blending. These chitosan coatings protect the gold nanoparticles, avoiding irreversible coalescence of the bare Au NPs and improving 


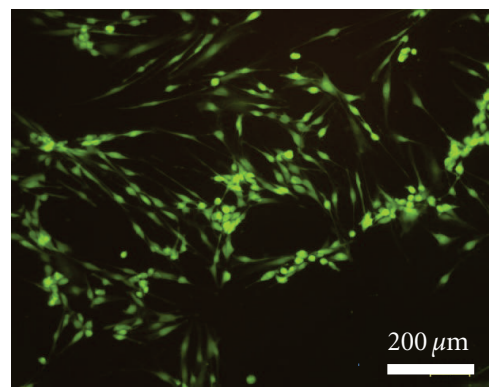

(a) control

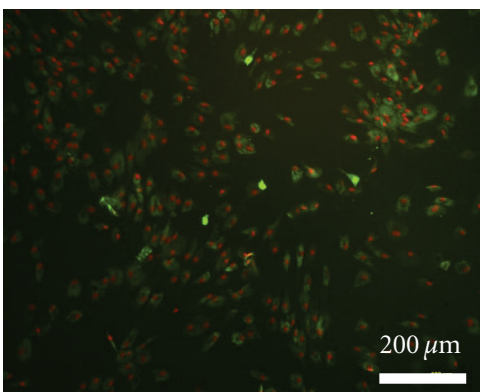

(d) $\mathrm{Au} / \mathrm{CS} \mathrm{NP}$

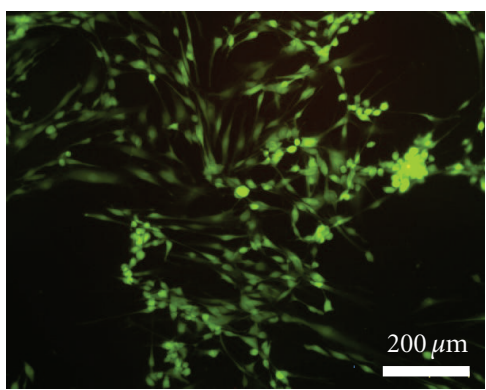

(b) without Au NP

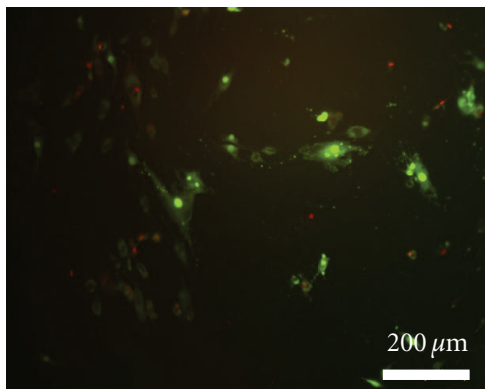

(e) $\mathrm{Au} / \mathrm{CMCS} N \mathrm{~N}$

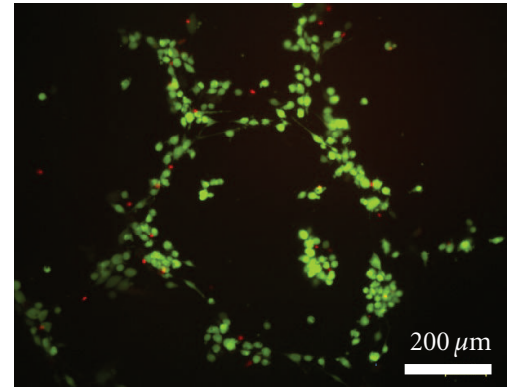

(c) bare Au NP

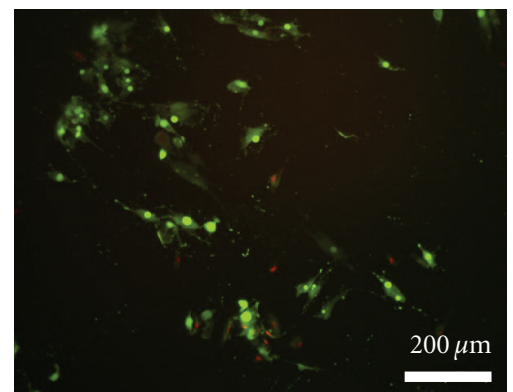

(f) $\mathrm{Au} /(\mathrm{CS}+\mathrm{CMCS}) \mathrm{NP}$

FIgURE 6: Live/dead stain images of the HDF cells treated with different Au NPs after the laser ablation (5 W/ $\left.\mathrm{cm}^{2}, 2 \mathrm{~min}\right)$. (a) control cells with no laser exposure, (b) cells without NPs, (c) cells treated with bare Au NPs, (d) cells treated with Au/CS NPs, (e) cells treated with $\mathrm{Au} / \mathrm{CMCS} \mathrm{NPs}$, and (f) cells treated with Au/(CS+CMCS) NPs.

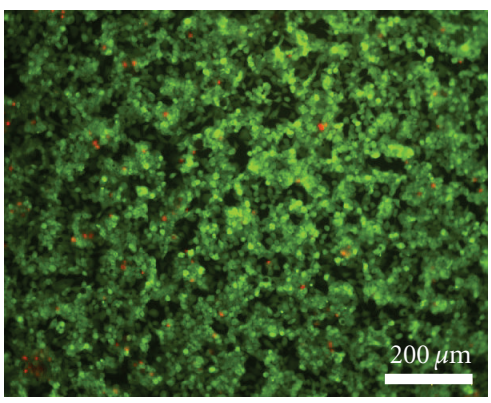

(a) control

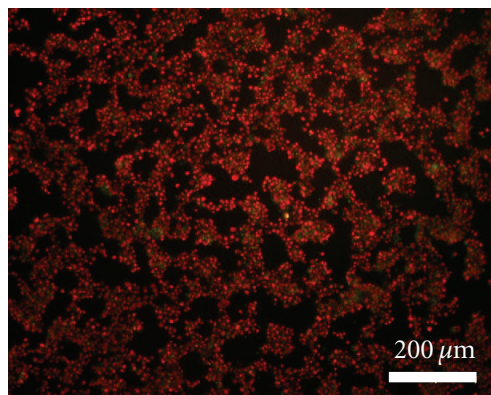

(d) $\mathrm{Au} / \mathrm{CS} \mathrm{NP}$

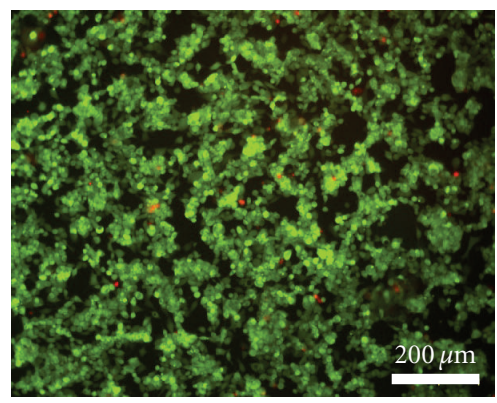

(b) without Au NP

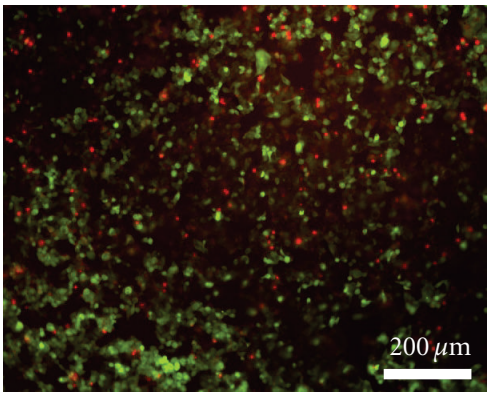

(e) $\mathrm{Au} / \mathrm{CMCS} N \mathrm{P}$

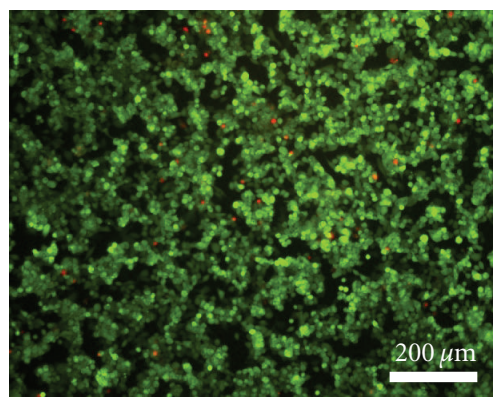

(c) bare Au NP

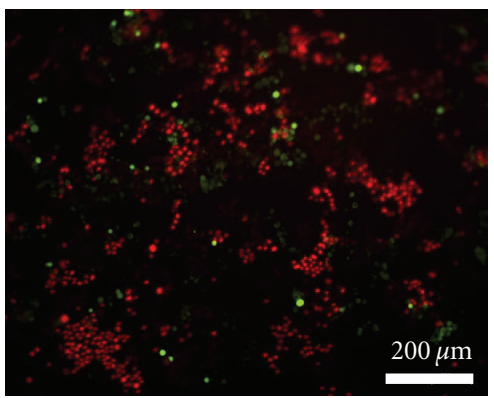

(f) $\mathrm{Au} /(\mathrm{CS}+\mathrm{CMCS}) \mathrm{NP}$

FIGURE 7: Live/dead stain images of the HepG2 cells treated with different Au NPs after the laser ablation (5 W/ $\left.\mathrm{cm}^{2}, 2 \mathrm{~min}\right)$. (a) control cells with no laser exposure, (b) cells without NPs, (c) cells treated with bare Au NPs, (d) cells treated with Au/CMCS NPs, (e) cells treated with $\mathrm{Au} / \mathrm{CS}$ NPs, and (f) cells treated with Au/(CS+CMCS) NPs. 


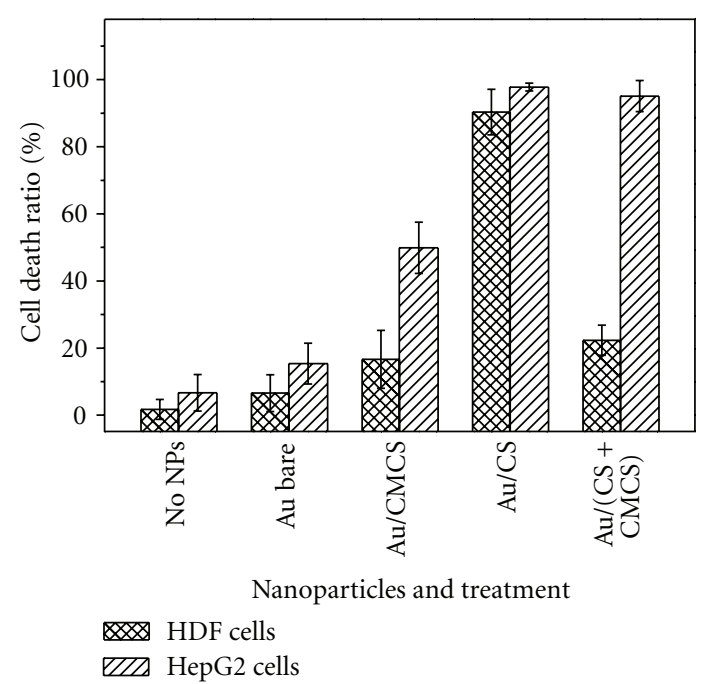

FIGURE 8: Death percentage of HDF cell and HepG2 cell cells treated with different $\mathrm{Au}$ NPs after the laser ablation at $5 \mathrm{~W} / \mathrm{cm}^{2}, 2 \mathrm{~min}$. (Average \pm STD, $n=3$ ).

their optical stability, making photothermal ablation possible. The surface charge of the Au/chitosan nanocomposites can be modified by adjusting the CS and CMCS components on the gold surface to achieve suitable binding of the $\mathrm{Au}$ NPs on the cell surface. The Au/CS nanocomposites show high accumulation on the cell surface and present the highest laser ablation efficiency. The CS and CMCS blended coatings allow $\mathrm{Au}$ NPs uniformly binding to the cells, showing higher selectivity between the cancer cells and normal cells during the photothermal ablation. These novel gold/polymer nanocomposites have great potential for photothermal therapy, due to low-dosage particle injection, lowpower NIR laser radiation, and quick ablation. More broad clinical relevance can be envisioned, such as the breast cancer treatment, skin cancer treatment, esophageal cancer treatment, and laryngocarcinoma cancer treatment.

\section{Acknowledgments}

Authors greatly acknowledge Deborah Hickman and Dr. Andrea Gobin for help with experiments and sample characterization. Research is supported by grants from Wallance Coulter Foundation: Early Career Phase 1 Award and the School of Medicine Summer Research Scholar Program.

\section{References}

[1] A. K. Gupta and M. Gupta, "Synthesis and surface engineering of iron oxide nanoparticles for biomedical applications," Biomaterials, vol. 26, no. 18, pp. 3995-4021, 2005.

[2] R. Shenhar, T. B. Norsten, and V. M. Rotello, "Polymermediated nanoparticle assembly: structural control and applications," Advanced Materials, vol. 17, no. 6, pp. 657-669, 2005.

[3] L. R. Hirsch, J. B. Jackson, A. Lee, N. J. Halas, and J. L. West, "A whole blood immunoassay using gold nanoshells," Analytical Chemistry, vol. 75, no. 10, pp. 2377-2381, 2003.
[4] D. S. Grubisha, R. J. Lipert, H. Y. Park, J. Driskell, and M. D. Porter, "Femtomolar detection of prostate-specific antigen: an immunoassay based on surface-enhanced raman scattering and immunogold labels," Analytical Chemistry, vol. 75, no. 21, pp. 5936-5943, 2003.

[5] T. Niidome, M. Yamagata, Y. Okamoto et al., "PEG-modified gold nanorods with a stealth character for in vivo applications," Journal of Controlled Release, vol. 114, no. 3, pp. 343347, 2006.

[6] M. C. Daniel and D. Astruc, "Gold nanoparticles: assembly, supramolecular chemistry, quantum-size-related properties, and applications toward biology, catalysis, and nanotechnology," Chemical Reviews, vol. 104, no. 1, pp. 293-346, 2004.

[7] A. M. Gobin, M. H. Lee, N. J. Halas, W. D. James, R. A. Drezek, and J. L. West, "Near-infrared resonant nanoshells for combined optical imaging and photothermal cancer therapy," Nano Letters, vol. 7, no. 7, pp. 1929-1934, 2007.

[8] C. Alric, J. Taleb, G. Le Duc et al., "Gadolinium chelate coated gold nanoparticles as contrast agents for both X-ray computed tomography and magnetic resonance imaging," Journal of the American Chemical Society, vol. 130, no. 18, pp. 5908-5915, 2008.

[9] X. Huang, P. K. Jain, I. H. El-Sayed, and M. A. El-Sayed, "Plasmonic photothermal therapy (PPTT) using gold nanoparticles," Lasers in Medical Science, vol. 23, no. 3, pp. 217-228, 2008.

[10] P. R. Stauffer, "Evolving technology for thermal therapy of cancer," International Journal of Hyperthermia, vol. 21, no. 8, pp. 731-744, 2005.

[11] S. N. Goldberg, "Radiofrequency tumor ablation: principles and techniques," European Journal of Ultrasound, vol. 13, no. 2, pp. 129-147, 2001.

[12] R. Valdagni, M. Amichetti, and G. Pani, "Radical radiation alone versus radical radiation plus microwave hyperthermia for $\mathrm{N}_{3}$ (TNM-UICC) neck nodes: a prospective randomized clinical trial," International Journal of Radiation Oncology Biology Physics, vol. 15, no. 1, pp. 13-24, 1988.

[13] R. Hergt, S. Dutz, R. Müller, and M. Zeisberger, "Magnetic particle hyperthermia: nanoparticle magnetism and materials development for cancer therapy," Journal of Physics Condensed Matter, vol. 18, no. 38, pp. S2919-S2934, 2006.

[14] W. Fink, H. van der Piepen, and W. Schneider, "Optical surface plasmon resonance as a measurement tool," pp. 419-423, 1975.

[15] I. H. El-Sayed, X. Huang, and M. A. El-Sayed, "Selective laser photo-thermal therapy of epithelial carcinoma using antiEGFR antibody conjugated gold nanoparticles," Cancer Letters, vol. 239, no. 1, pp. 129-135, 2006.

[16] X. Huang, I. H. El-Sayed, W. Qian, and M. A. El-Sayed, "Cancer cell imaging and photothermal therapy in the nearinfrared region by using gold nanorods," Journal of the American Chemical Society, vol. 128, no. 6, pp. 2115-2120, 2006.

[17] M. Hu, H. Petrova, J. Chen et al., "Ultrafast laser studies of the photothermal properties of gold nanocages," Journal of Physical Chemistry B, vol. 110, no. 4, pp. 1520-1524, 2006.

[18] G. Zhang, J. B. Jasinski, J. L. Howell, D. Patel, D. P. Stephens, and A. M. Gobin, "Tunability and stability of gold nanoparticles obtained from chloroauric acid and sodium thiosulfate reaction," Nanoscale Research Letters, vol. 7, article 337, 2012.

[19] Y. Shigemasa and S. Minami, "Applications of chitin and chitosan for biomaterials," Biotechnology and Genetic Engineering Reviews, vol. 13, pp. 383-420, 1996. 
[20] M. J. Laudenslager, J. D. Schiffman, and C. L. Schauer, "Carboxymethyl chitosan as a matrix material for platinum, gold, and silver nanoparticles," Biomacromolecules, vol. 9, no. 10, pp. 2682-2685, 2008.

[21] I. Wedmore, J. G. McManus, A. E. Pusateri, and J. B. Holcomb, "A special report on the chitosan-based hemostatic dressing: experience in current combat operations," Journal of TraumaInjury, Infection and Critical Care, vol. 60, no. 3, pp. 655-658, 2006.

[22] X. Sun, G. Zhang, D. Patel, D. Stephens, and A. M. Gobin, "Targeted cancer therapy by immunoconjugated gold-gold sulfide nanoparticles using protein $\mathrm{G}$ as a cofactor," Annals of Biomedical Engineering, vol. 40, no. 10, pp. 2131-2139, 2012.

[23] X. G. Chen and H. J. Park, "Chemical characteristics of Ocarboxymethyl chitosans related to the preparation conditions," Carbohydrate Polymers, vol. 53, no. 4, pp. 355-359, 2003.

[24] Y. Y. Liang and L. M. Zhang, "Bioconjugation of papain on superparamagnetic nanoparticles decorated with carboxymethylated chitosan," Biomacromolecules, vol. 8, no. 5, pp. 14801486, 2007.

[25] G. Zhang, J. B. Jasinski, D. Patel, K. James, X. Sun, and A. M. Gobin, "Gold nanoparticles with tuning near infrared absorption via reaction of $\mathrm{HAuCl}_{4}$ and $\mathrm{Na}_{2} \mathrm{~S}_{2} \mathrm{O}_{3}$ for low power photothermal cancer therapy," MRS Procedings, vol. 1416, 2012.

[26] A. M. Gobin, E. M. Watkins, E. Quevedo, V. L. Colvin, and J. L. West, "Near-infrared-resonant gold/gold sulfide nanoparticles as a photothermal cancer therapeutic agent," Small, vol. 6, no. 6, pp. 745-752, 2010.

[27] H. Huang and X. Yang, "Synthesis of chitosan-stabilized gold nanoparticles in the absence/presence of tripolyphosphate," Biomacromolecules, vol. 5, no. 6, pp. 2340-2346, 2004.

[28] D. S. Dos Santos, P. J. G. Goulet, N. P. W. Pieczonka, O. N. Oliveira, and R. F. Aroca, "Gold nanoparticle embedded, selfsustained chitosan films as substrates for surface-enhanced Raman scattering," Langmuir, vol. 20, no. 23, pp. 1027310277, 2004.

[29] P. R. Selvakannan, S. Mandal, S. Phadtare, R. Pasricha, and M. Sastry, "Capping of gold nanoparticles by the amino acid lysine renders them water-dispersible," Langmuir, vol. 19, no. 8, pp. 3545-3549, 2003.

[30] K. H. Su, Q. H. Wei, X. Zhang, J. J. Mock, D. R. Smith, and S. Schultz, "Interparticle coupling effects on plasmon resonances of nanogold particles," Nano Letters, vol. 3, no. 8, pp. 10871090, 2003.

[31] S. K. Ghosh and T. Pal, "Interparticle coupling effect on the surface plasmon resonance of gold nanoparticles: from theory to applications," Chemical Reviews, vol. 107, no. 11, pp. 47974862, 2007.

[32] E. Guibal, "Interactions of metal ions with chitosan-based sorbents: a review," Separation and Purification Technology, vol. 38, no. 1, pp. 43-74, 2004.

[33] Z. P. Zhao, Z. Wang, and S. C. Wang, "Formation, charged characteristic and BSA adsorption behavior of carboxymethyl chitosan/PES composite MF membrane," Journal of Membrane Science, vol. 217, no. 1-2, pp. 151-158, 2003. 

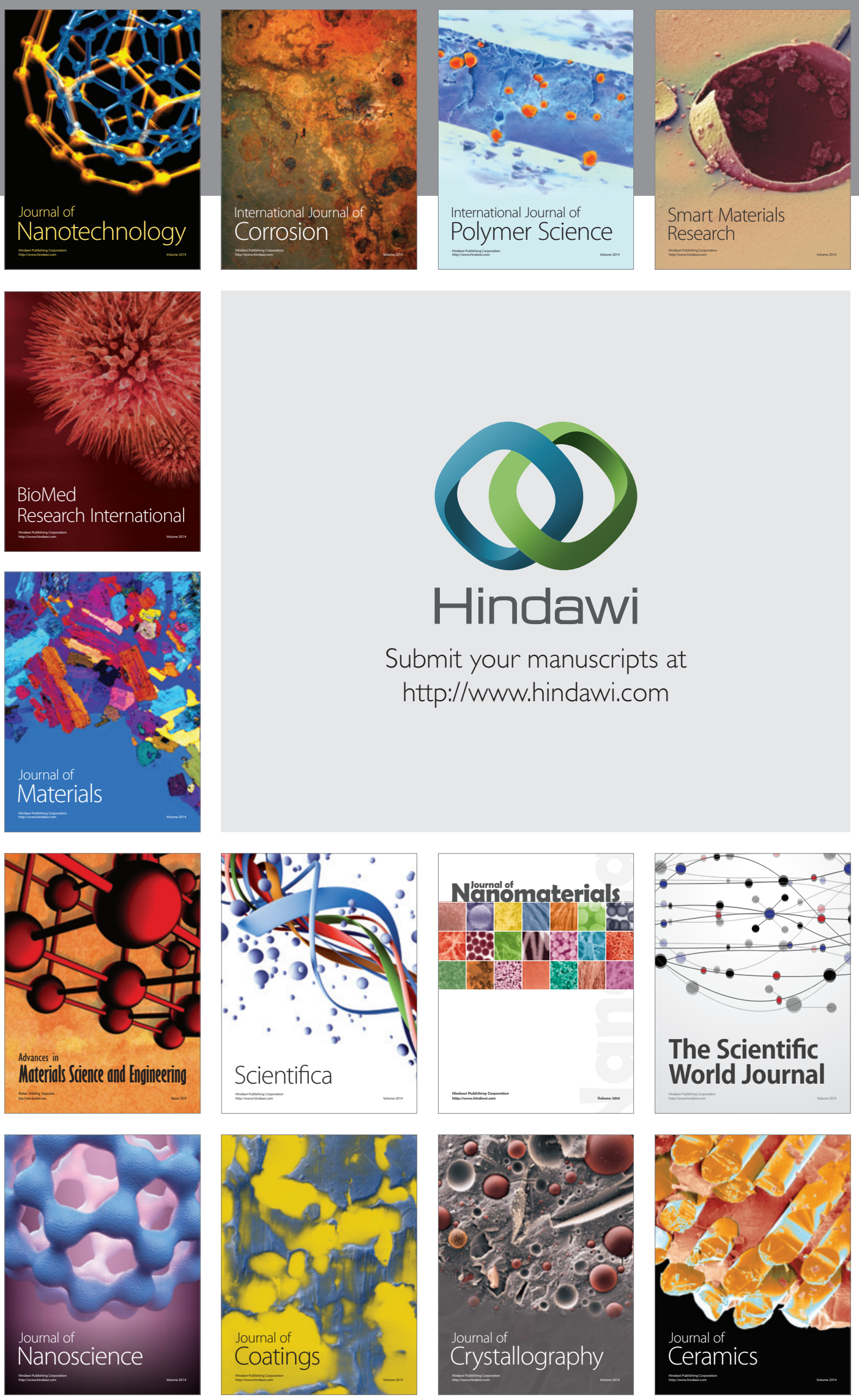

The Scientific World Journal

Submit your manuscripts at

http://www.hindawi.com

\section{World Journal}

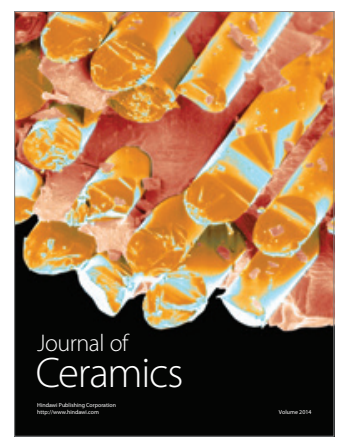

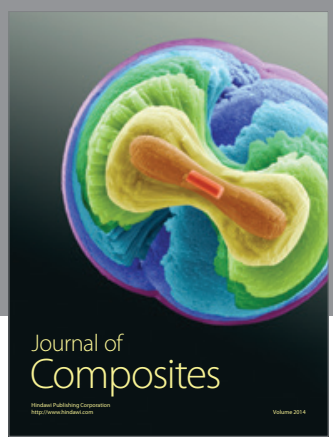
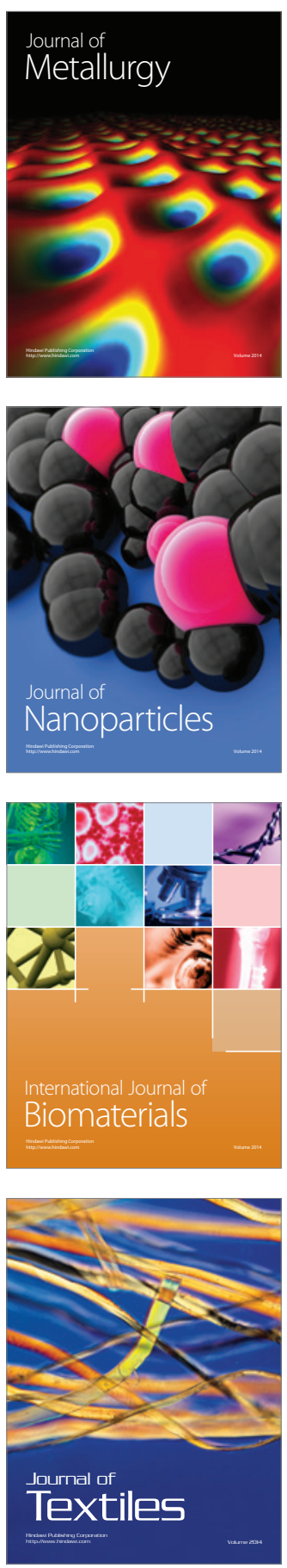